\title{
In vitro antioxidant activity and in vivo antifatigue effect of layered double hydroxide nanoparticles as delivery vehicles for folic acid
}

This article was published in the following Dove Press journal:

International Journal of Nanomedicine

4 December 2014

Number of times this article has been viewed

\author{
Lili Qin ${ }^{1, *}$ \\ Wenrui Wang 2 ,* \\ Songhui You' \\ Jingmei Dong' \\ Yunhe Zhou' \\ Jibing Wang' \\ 'Department of Physical Education, \\ ${ }^{2}$ School of Life Science and \\ Technology, Tongji University, Shanghai, \\ People's Republic of China \\ *These authors contributed equally \\ to this work
}

Correspondence: Lili Qin

Department of Physical Education, Tongji University, 1239 Siping Road, Shanghai

200092, People's Republic of China

Tel +86 2I 659817 II

Fax +86 21 65983777

Email qinlili@tongji.edu.cn
Abstract: Folic acid antioxidants were successfully intercalated into layered double hydroxides (LDH) nanoparticles according to a previous method with minor modification. The resultant folic acid-LDH constructs were then characterized by X-ray powder diffraction and transmission electron microscopy. The in vitro antioxidant activities, cytotoxicity effect, and in vivo antifatigue were examined by a series of assays. The results showed that folic acid-LDH antioxidant system can scavenge 1,1-diphenyl-2-picrylhydrazyl and hydroxyl free radicals and chelate pro-oxidative $\mathrm{Cu}^{2+}$. The in vitro cytotoxicity assays indicated that folic acid-LDH antioxidant system had no significant cytotoxic effect or obvious toxicity to normal cells. It also prolonged the forced swimming time of the mice by $32 \%$ and $51 \%$ compared to folic acid and control groups, respectively. It had an obvious effect on decreasing the blood urea nitrogen and blood lactic acid, while increasing muscle and hepatic glycogen levels. Therefore, folic acid-LDH might be used as a novel antioxidant and antifatigue nutritional supplement.

Keywords: antioxidant supplementation, free radicals, biomaterials, drug delivery

\section{Introduction}

The classic "radical theory" indicates that intense exercise can produce an imbalance between the body's oxidation and antioxidation systems. The accumulation of reactive free radicals will lead to oxidative stress which can cause oxidative damage to bimolecular such as protein, lipid, and DNA. ${ }^{1}$ It also can induce injury to tissues or organs and is one of the reasons for fatigue. ${ }^{2,3}$ Fatigue is a physiological phenomenon that arises from physical stress or exhaustive exercises leading to the reduction of physical endurance capacity. ${ }^{4}$ Therefore, supplementing with antioxidants has been considered to have an important role in reducing the degree of fatigue caused by free radical and oxidative stress. ${ }^{5}$

Folic acid is a vitamin of the B group. It can be converted into a coenzyme N5,N10-dimethylene-5,6,7,8-tetrahydrofolate in vivo through a series of enzymatic transformations. It has purine and pyrazine type rings and two amino acids on the pyrazine ring. Its lactim form has a hydroxyl group on the purine type ring. When folic acid reacts with oxidizing free radicals, this hydroxyl group can play an important role in inhibiting the oxidation effect. ${ }^{6}$ Therefore, some researches have reported that folic acid possesses a significant antioxidant effect and reduces oxidative stress. ${ }^{7,8}$ However, folic acid, like other natural antioxidants, is limited by poor stability, short half-life in vivo, low bioavailability, and is easily degraded by proteolytic or gastrointestinal enzymes. Therefore, using nanomaterials as an efficient delivery system for antioxidants can solve these problems and improve clinical therapy. 
Layered double hydroxides (LDHs) as one of the inorganic delivery nanoparticles have recently been receiving considerable attentions. ${ }^{9,10}$ They possess some promising advantages such as low cost, good biocompatibility, low toxicity to mammalian cells, controlled release property, and full protection for loaded drugs. Many LDH compounds intercalated with beneficial organic anions such as DNA, ${ }^{11,12}$ amino acids, ${ }^{13}$ anionic polymers ${ }^{14}$ pesticides, ${ }^{15}$ and drugg ${ }^{16-19}$ have been prepared successfully. Furthermore, the antioxidant intercalated into LDH delivery can be used as a nanomaterial antioxidant supplementation, thereby expanding its range of applications.

In our previous research, we reported that folic acidLDH nanohybrids could be used as a reservoir of folic acid molecules. ${ }^{20}$ In the present study, folic acid-LDH was prepared according to a previous method with some modifications. Our goal here is to examine the in vitro antioxidative capacity of folic acid-LDH as well as its in vivo antifatigue effects.

\section{Materials and methods \\ Materials}

$\mathrm{Mg}\left(\mathrm{NO}_{3}\right)_{2} \cdot 6 \mathrm{H}_{2} \mathrm{O}, \mathrm{Al}\left(\mathrm{NO}_{3}\right)_{3} \cdot 9 \mathrm{H}_{2} \mathrm{O}$, folic acid, and 1,1-diphenyl2-picrylhydrazyl (DPPH) were purchased from China National Medicine Group, Shanghai Chemical Reagents Company (Shanghai, People's Republic of China), and were used without further purification. RPMI-1640, fetal calf serum, penicillin G, streptomycin, and trypsin were obtained from GIBCO BRL (Grand Island, NY, USA). Dimethyl sulfoxide and [3-(4,5-dimethylthiazol-2-yl)-2, 5-diphenyltetrazolium bromide] (MTT) were purchased from Sigma-Aldrich Co. (St Louis, MO, USA). Deionized water was decarbonated by boiling before use in all applications.

\section{Animals}

All the in vivo tests were carried by Academy of Experimental Animal Center of Tongji University (Shanghai, People's Republic of China), which obtained the permission for performing the research protocols and all animal experiments were approved by the ethics committee of Tongji University. Sixty male Kunming mice (15-22 g), specific pathogen-free grade, were purchased from Shanghai Laboratory Animal Centre (SLAC). Animals were allowed to adapt to their surroundings for 1 week before starting the experiments. Mice were housed five to six per cage at room temperature $\left(22^{\circ} \mathrm{C} \pm 2{ }^{\circ} \mathrm{C}\right)$ and moderate humidity $(50 \% \pm 10 \%)$, with a $12-/ 12$-hour light/dark cycle. The noise was $<60 \mathrm{~dB}$. They were fed a balanced murine diet with drinking water available ad libitum provided by the Academy of Experimental Animal Center of Tongji University.
Sixty mice were randomly divided into six groups with ten mice each: two control groups, two folic acid treatment groups, and two folic acid-LDH treatment groups. The control groups were administered distilled water at $10 \mathrm{~mL} / \mathrm{kg}$ of body weight. The other groups were administered folic acid at $5 \mathrm{mg} / \mathrm{kg}$ of body weight. The amount of folic acid was adjusted to be equal to that of the folic acid-LDH treatment group. Water, folic acid, and folic acid-LDH were administered for 28 days. The forced swimming test was conducted on the last day and corresponding biochemical parameters were measured.

\section{Preparation of folic acid-LDH}

Folic acid-LDH was prepared according to a previous coprecipitation method with some modifications. ${ }^{20} \mathrm{~A}$ mixed solution $(40 \mathrm{~mL})$ of $0.17 \mathrm{M} \mathrm{NaOH}$ and $0.1 \mathrm{M}$ folic acid was stirred at room temperature for 10 minutes, and then a solution $(10 \mathrm{~mL})$ containing $0.3 \mathrm{M} \mathrm{Mg}\left(\mathrm{NO}_{3}\right)_{2}$ and $0.1 \mathrm{M}$ $\mathrm{Al}\left(\mathrm{NO}_{3}\right)_{3}$ was added. The mixture was continuously vigorously magnetically stirred under $\mathrm{N}_{2}$ for 3 hours. The resultant precipitates were centrifuged (4,500 rpm, 15 minutes) and washed twice with deionized water, and then dispersed in $40 \mathrm{~mL}$ of double distilled water and treated in an autoclave at $50^{\circ} \mathrm{C}$ for 12 hours. The LDH solid was harvested by filtering and vacuum drying overnight. The product was denoted as folic acid-LDH.

\section{Characterization}

$\mathrm{X}$-ray powder diffraction (XRD) patterns were recorded on a Rigaku Miniflex diffractometer using $\mathrm{CuKa}$ radiation $\left(\lambda=0.154060 \mathrm{~nm}, 40 \mathrm{kV}, 40 \mathrm{~mA}\right.$, step of $\left.0.0330^{\circ}\right)$. The average particle size ( $z$-average size) and size distribution were measured using photon correlation spectroscopy (PCS) (LS230; Beckman Coulter) at $25^{\circ} \mathrm{C}$ under a fixed angle of $90^{\circ}$ in disposable polystyrene cuvettes. The measurements were recorded using a $\mathrm{He}-\mathrm{Ne}$ laser of $633 \mathrm{~nm}$. Zeta potential distribution and polydispersity index of the nanoparticles were analyzed by Nano ZS (Malvern Instruments, Malvern, UK). Transmission electron micrographs were taken using a JEOL (Tokyo, Japan) 1230 transmission electron microscope (TEM). Ultraviolet (UV)/visible (VIS) absorption spectra were measured on a CARY 50 spectrophotometer.

\section{In vitro antioxidant activities of folic acid-LDH}

Hydroxyl radical scavenging activity assay

The hydroxyl radical scavenging activity assay was performed according to the method by de Avellar et al with some 
modifications. ${ }^{21}$ The solution contained 1,10-phenanthroline $(5.0 \mathrm{mM}, 600 \mu \mathrm{L}), \mathrm{FeSO}_{4}(5.0 \mathrm{mM}, 600 \mu \mathrm{L})$, and ethylenediaminetetraacetic acid $(15 \mathrm{mM}, 600 \mu \mathrm{L})$ dissolved in sodium phosphate buffer $\left(0 \times 10^{-3} \mathrm{mg} / \mathrm{mL}\right.$ to $\left.20 \times 10^{-3} \mathrm{mg} / \mathrm{mL}\right)$. Then, folic acid, folic acid-LDH $\left(0-20 \times 10^{-3} \mathrm{mg} / \mathrm{mL}\right)$, and $\mathrm{H}_{2} \mathrm{O}_{2}$ $(0.01 \%)$ were added. The mixture was incubated at $37^{\circ} \mathrm{C}$ for 60 minutes, and the absorbance was measured by UV/VIS absorption spectra on a CARY 50 spectrophotometer at $536 \mathrm{~nm}$. The result was determined using the following equation:

$$
\begin{aligned}
& \text { Hydroxyl radical } \\
& \text { scavenging activity }(\%)
\end{aligned}=\frac{\left(A_{S}-A_{0}\right) \times 100}{A_{C}-A_{0}}
$$

where $A S$ is the absorbance of the sample; $A_{0}$ is the absorbance of the blank solution using distilled water instead of sample; and $A C$ is the absorbance of a control solution in the absence of $\mathrm{H}_{2} \mathrm{O}_{2}$.

\section{DPPH radical scavenging activity assay}

The scavenging activity of folic acid-LDH on DPPH free radicals was measured according to the method of Shimada et al with minor modification. ${ }^{22}$ Two milliliters of folic acid and folic acid-LDH $\left(0 \times 10^{-3} \mathrm{mg} / \mathrm{mL}\right.$ to $\left.6 \times 10^{-3} \mathrm{mg} / \mathrm{mL}\right)$ were mixed with $2.0 \mathrm{~mL}$ of $0.10 \mathrm{mM}$ DPPH that was dissolved in $95 \%$ ethanol. The mixture was then shaken vigorously and kept for 30 minutes in the dark at room temperature. The absorbance of the resulting solution was recorded at $517 \mathrm{~nm}$. A lower absorbance represents a higher DPPH scavenging activity. The scavenging activity was calculated using the following equation:

Scavenging activity $(\%)=\frac{\left(A_{D P P H \text { sample }}-A_{\text {Sample control }}\right) \times 100}{A_{D P P H \text { blank }}}$

here, $A D P P H_{\text {sample }}$ is the value for the $2 \mathrm{~mL}$ of sample solution mixed with the DPPH solution; ASample ${ }_{\text {control }}$ is the value for the $2 \mathrm{~mL}$ of sample solution mixed with the $2 \mathrm{~mL}$ of $95 \%$ ethanol; and ADPPH blank is the value for the $2 \mathrm{~mL}$ of $95 \%$ ethanol mixed with the DPPH solution.

\section{Cupric ion chelating activity assay}

The chelating effect of folic acid-LDH on pro-oxidative $\mathrm{Cu}^{2+}$ was investigated according to Zhu et al. ${ }^{23}$ In the test, $1 \mathrm{~mL}$ of $2 \mathrm{mM} \mathrm{CuSO}_{4}$ was mixed with $1 \mathrm{~mL}$ of pyridine ( $\mathrm{pH} 7.0$ ) and $0.1 \%$ pyrocatechol violet. After the addition of $1 \mathrm{~mL}$ of folic acid and folic acid-LDH $\left(0-6 \times 10^{-3} \mathrm{mg} / \mathrm{mL}\right)$, the blue color disappeared because of the dissociation of $\mathrm{Cu}^{2+}$. After reacting for 5 minutes, it was recorded by measuring the absorbance at $632 \mathrm{~nm}$. An equivalent volume of distilled water instead of the sample was used for the blank. The $\mathrm{Cu}^{2+}$ chelating activity were calculated as:

$$
\text { Cu chelating activity }(\%)=\frac{A_{0}-A_{S}}{A_{0}} \times 100 \%
$$

where $A S$ is the absorbance of the sample and $A_{0}$ is the absorbance of the blank solution using distilled water instead of sample.

\section{In vitro cytotoxicity effect of folic acid-LDH}

GES-1 cells derived from human gastric mucosa epithelium were obtained from Shanghai Institute of Biochemistry and Cell Biology (Shanghai, People's Republic of China). Cells were routinely cultured at $37^{\circ} \mathrm{C}$ in $5 \% \mathrm{CO}_{2}$ atmosphere in the flasks containing $10 \mathrm{~mL}$ of Dulbecco's Modified Eagle's Medium supplemented with 10\% fetal calf serum, $100 \mathrm{U} / \mathrm{mL}$ penicillin, and $100 \mu \mathrm{g} / \mathrm{mL}$ streptomycin. At 80\%-90\% confluence, cells were disassociated using trypsinethylenediaminetetraacetic acid and plated at a density of approximately $2 \times 10^{4}$ cells per well in a 96 -well plate. The number of viable cells was determined by MTT assay with 3-(4,5-dimethylthiazole-2-yl)-2,5-phenyltetrazolium bromide. After incubation at $37^{\circ} \mathrm{C}$ in a $5 \% \mathrm{CO}_{2}$ humid atmosphere for 24 hours, triplicate wells were treated with folic acid, folic acid-LDH, and $\mathrm{LDH}$ of various concentrations $(5,10,20$, and $40 \mu \mathrm{g} / \mathrm{mL})$. The control cultures were grown in growth medium only. Then, the incubation was continued as indicated above for 4 hours. For the LDH-treated wells, the amount of LDH was adjusted to be equal to that of folic acid-LDH-treated wells. Subsequently, each well was incubated with $20 \mu \mathrm{L}(5 \mathrm{mg} / \mathrm{mL})$ of MTT dye solution for 4 hours at $37^{\circ} \mathrm{C}$. After removal of the MTT solution, cells were treated with $150 \mu \mathrm{L}$ dimethyl sulfoxide and the absorbance at $490 \mathrm{~nm}$ was quantified using a microplate reader (ELX 800 UV; BIO-TEK, Vermont, USA). Cell viability was calculated by means of the following formula:

$$
\text { Cell viability }(\%)=\frac{O D_{490(\text { sample })}-O D_{490(\text { blank })}}{O D_{490(\text { control })}-O D_{490(\text { blank })}} \times 100 \%
$$

\section{In vivo antifatigue effect of folic acid-LDH Effect on swimming time to fatigue}

After 28 days, ten mice were taken from each group for the forced swimming test. The procedure was used according to the previous study with some modification. ${ }^{24}$ Briefly, 
30 minutes after the final treatment with folic acid, folic acidLDH or distilled water, the mice were placed individually in a swimming pool $(50 \times 50 \times 40 \mathrm{~cm})$ filled with water to a depth of $30 \mathrm{~cm}$ and the water temperature was kept at $25^{\circ} \mathrm{C} \pm 1^{\circ} \mathrm{C}$. A lead sinker (10\% of the body weight) was attached to the tail root of each mouse. The mice were assessed to be exhausted when they failed to rise to the surface within 10 seconds. The swimming time was recorded immediately. In the last stage, the mice were then removed from the water, dried with a paper towel, and returned to their original cages. The pool water was replaced after each session. The swimming time to exhaustion was used as the index of the forced swimming capacity.

\section{Determination of biochemical parameters related to fatigue}

After the final treatment with folic acid, folic acid-LDH or distilled water, the mice were allowed to rest for 30 minutes. Then they were placed in the swimming tank. After swimming for 30 minutes without loads, the whole blood was collected via retro-orbital venipuncture, and serum was prepared by centrifugation at $4,000 \mathrm{rpm}$ at $4^{\circ} \mathrm{C}$ for 15 minutes. Then, the content of blood urea nitrogen (BUN) and blood lactic acid (BLA) were determined. The liver and gastrocnemius muscle were collected to be made into $10 \%$ homogenates with saline as soon as possible. All of the biochemical parameters were determined using the commercial diagnostic kits according to the procedures provided by the Nanjing Jiancheng Bioengineering Institute.

\section{Statistical analysis}

For statistical analysis, the data were presented as the mean \pm standard deviation of three independent experiments. One-way analysis of variance followed with $t$-test was performed using the SPSS program, and the levels of significance were represented in each result.

\section{Results and discussion}

\section{Preparation and characterization of folic acid-LDH}

The powder XRD patterns of folic acid-LDH sample was compared to the pristine LDH pattern (Figure 1). The patterns of LDH and folic acid-LDH are typical of lamellar materials. They are characterized with basal reflections, associated with harmonics at low $2 \theta$ angles, and weaker nonbasal reflections at higher angles. ${ }^{25}$ As shown in Figure 1A, the pristine LDH is well crystallized, showing a typical XRD pattern for LDH-NO $\mathrm{N}_{3}$ with the sharp (003) and (006) basal peaks. The

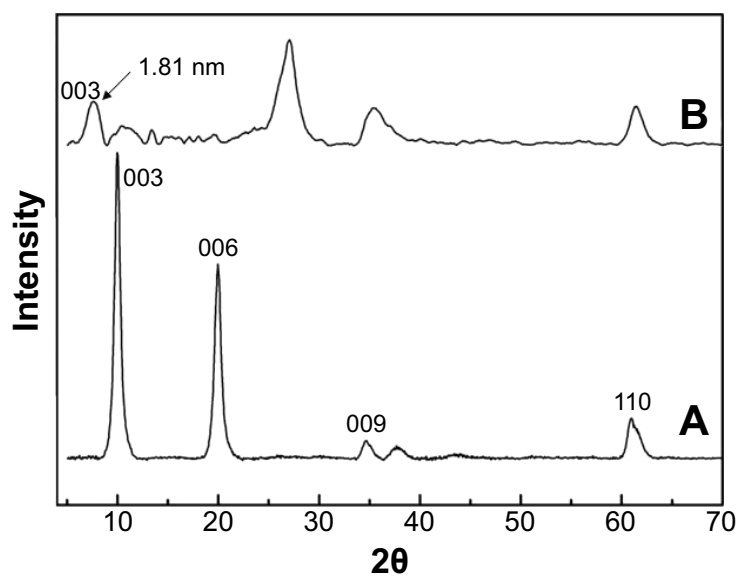

Figure I Powder X-ray diffraction patterns for LDH and folic acid-LDH. Notes: (A) LDH and (B) folic acid-LDH. The pointed arrow indicates the new basal diffractions attributed to the folic acid intercalated into LDH phase.

Abbreviation: LDH, layered double hydroxide.

intercalation of folic acid can lead to a significant increase in the interlayer space. For the pattern of folic acid-LDH in Figure 1B, the reflection peaks shift to lower angles with becoming weaker and broader. These reflections could be attributed to folic acid intercalation to LDH layer with the basal spacing from 0.88 to $1.18 \mathrm{~nm}$ (Figure 2). Assuming a thickness of $0.48 \mathrm{~nm}$ for the brucite-like layer of LDH, the gallery height in folic acid-LDH became $0.70 \mathrm{~nm}$, which is shorter than the molecular length of folic acid $(\sim 2.15 \mathrm{~nm}){ }^{20,26}$ The reason for the discrepancy between the calculated gallery height $(0.70 \mathrm{~nm})$ and estimated molecular length $(\sim 2.15 \mathrm{~nm})$ is the strong electrostatic interactions between folic acid anions and LDH hydroxide layers. It also can be deduced that the folic acid molecules are arranged in a tilted longitudinal monolayer, with tilting angles of approximately $70^{\circ}$. As listed in Table 1, the amount of folic acid intercalated into the LDH was calculated using a UV-VIS spectrophotometer and its drug loading was about $32.4 \%$ (w/w). Thus, in this study, the preparation procedure offers a much higher drug-loading amount compared to our previous study. ${ }^{20}$

\section{Physicochemical characteristics of folic acid-LDH}

TEM samples were prepared by adding $300 \mathrm{~mL}$ of $\mathrm{LDH}$ and folic acid-LDH slurry $(0.5 \mathrm{mg} / \mathrm{mL})$ to $15 \mathrm{~mL}$ of deionized water followed by probe sonication of the solution for 2 minutes. A drop of the solution was then placed on a holey carbon copper grid and air dried. As shown in Figure 3 (A and B), Mg/Al-LDH was observed as compact, nonporous crystallites with a hexagonal plate-like shape and a diameter of approximately $50 \mathrm{~nm}$. When the folic 


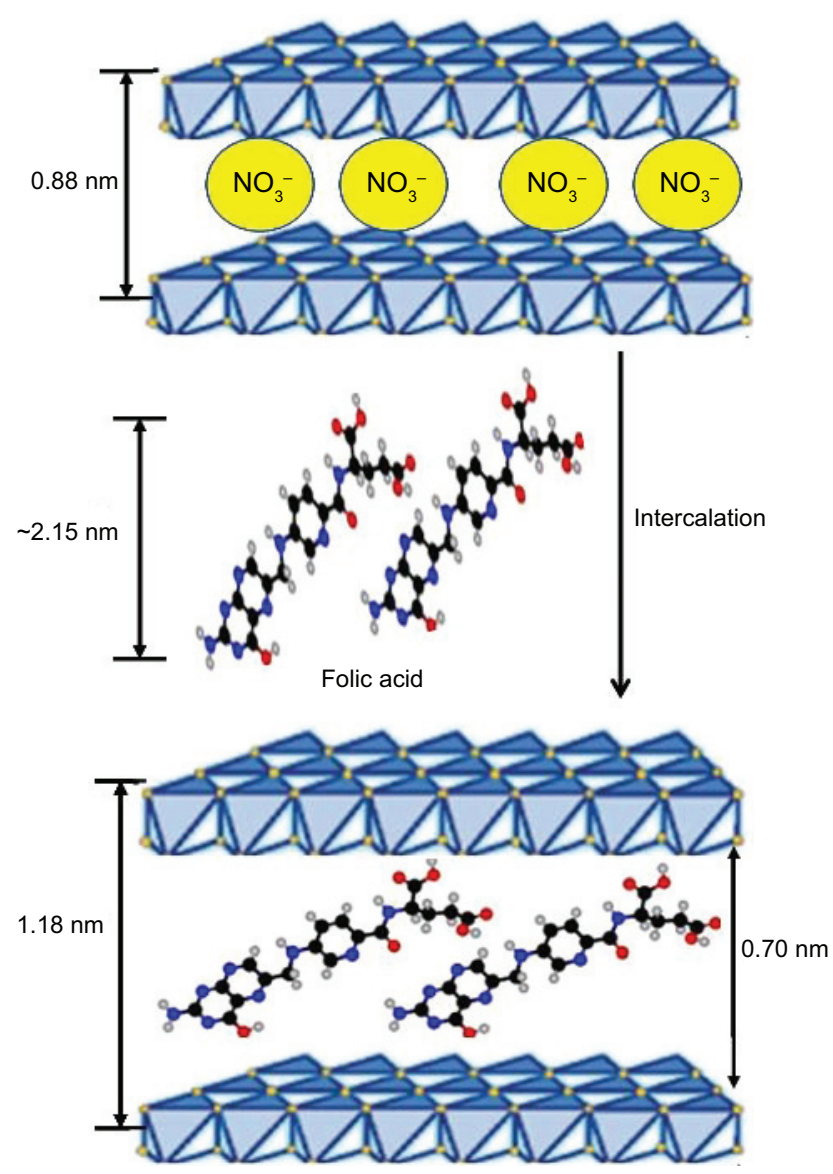

Figure 2 Schematic model of layered double hydroxide structure before and after folic acid intercalation.

acid intercalated into the inorganic host, the shape of the nanohybrid changed from regular hexagons to ellipses, and the crystal size was increased to a diameter range of 80-90 $\mathrm{nm}$. The particle size and distribution dynamics of folic acid-LDH were investigated by PCS. The mean particle size of folic acid-LDH is narrowly distributed with a nominal hydrodynamic diameter of $95.3 \mathrm{~nm}$ (Figure 3C), and the zeta potentials are $27.5 \mathrm{mV}$ with a polydispersity index of $0.317 \pm 0.005$ (Figure 3D). The size of particles measured by PCS is larger than those estimated using TEM. This is because PCS is used for hydrodynamic diameter determination, while TEM is used for characterization of particles in the dried state. ${ }^{27}$

Table I The $d$-values of nanohybrid and folic acid loading

\begin{tabular}{llllll}
\hline Nanohybrid & \multicolumn{2}{l}{ d-values, $\mathbf{n m}$} & Gallery & Folic acid \\
\cline { 2 - 4 } & $\boldsymbol{d}_{003}$ & $\boldsymbol{d}_{\mathbf{0 0 6}}$ & $\boldsymbol{d}_{110}$ & height/nm & loading, \% (w/w) \\
\hline LDH-NO $_{3}$ & 0.88 & 0.45 & 0.15 & 0.48 & - \\
Folic acid-LDH & I.I8 & 0.83 & 0.15 & 0.70 & 32.4 \\
\hline
\end{tabular}

Note: - indicates no data.

Abbreviation: LDH, layered double hydroxide.

\section{In vitro antioxidant activities of folic acid-LDH}

In vitro antioxidant activities of folic acid-LDH were evaluated by several antioxidant activity-determining assays based on different active mechanisms. The different free radical systems including hydroxyl radical, DPPH, and Cupric ion were employed in this study.

Hydroxyl radicals $(\mathrm{OH} \cdot)$ are one of reactive oxygen species generated in the human body. They are the most destructive reactive oxygen species and can be formed from superoxide anion and hydrogen peroxide in the presence of metal ions such as copper or iron. ${ }^{28}$ Due to their high level of reaction activity, they can induce oxidative damage to almost all important biomolecules including amino acids, DNA, polyunsaturated fatty acids, and proteins. This can lead to physiological disorders and disease. ${ }^{29}$ The hydroxyl radical scavenging ability of folic acid-LDH with different concentrations is shown in Figure 4. As the folic acid-LDH concentration increased, the hydroxyl radical scavenging activities exhibited an increasing tendency to higher scavenging activity. When the concentration reached $20 \times 10^{-3} \mathrm{mg} / \mathrm{mL}$, it showed $83.86 \%$ hydroxyl radical scavenging activity, which is obviously higher than that of folic acid.

The relatively stable DPPH radical was widely used to test the ability of compounds to act as free radical scavengers or hydrogen donors to determine the antioxidant activity. This method has been recommended as an easy and accurate method of measuring the antioxidant activity. ${ }^{30}$ Figure 5 shows the DPPH radical scavenging capacity of folic acid-LDH in comparison with folic acid as reference. The results suggest significant antioxidant activity in a concentration-dependent manner. Folic acid-LDH shows an excellent DPPH radical scavenging activity of $91.71 \%$, while folic acid offers $78.23 \%$ inhibitory effect at the concentration of $6 \times 10^{-3} \mathrm{mg} / \mathrm{mL}$.

The radical system used for antioxidant evaluation may influence the experimental results, so two or more radical systems are required to investigate the antioxidant capacities. ${ }^{31}$ The folic acid-LDH was analyzed for pro-oxidative $\mathrm{Cu}^{2+}$ chelating effect to better examine its antioxidant properties. As shown in Figure 6, both folic acid and folic-LDH suggest significant $\mathrm{Cu}^{2+}$ chelating activity in a concentrationdependent manner. In this assay, folic acid-LDH shows the highest chelating activity of $73.44 \%$ at the concentration of $6 \times 10^{-3} \mathrm{mg} / \mathrm{mL}$. On the other hand, folic acid shows about $65.89 \%$ chelating effect of pro-oxidative $\mathrm{Cu}^{2+}$.

In summary, firstly, all antioxidant activity assays indicate that folic acid does not lose its antioxidant properties after the intercalation into LDH layers. Secondly, it is evident that 

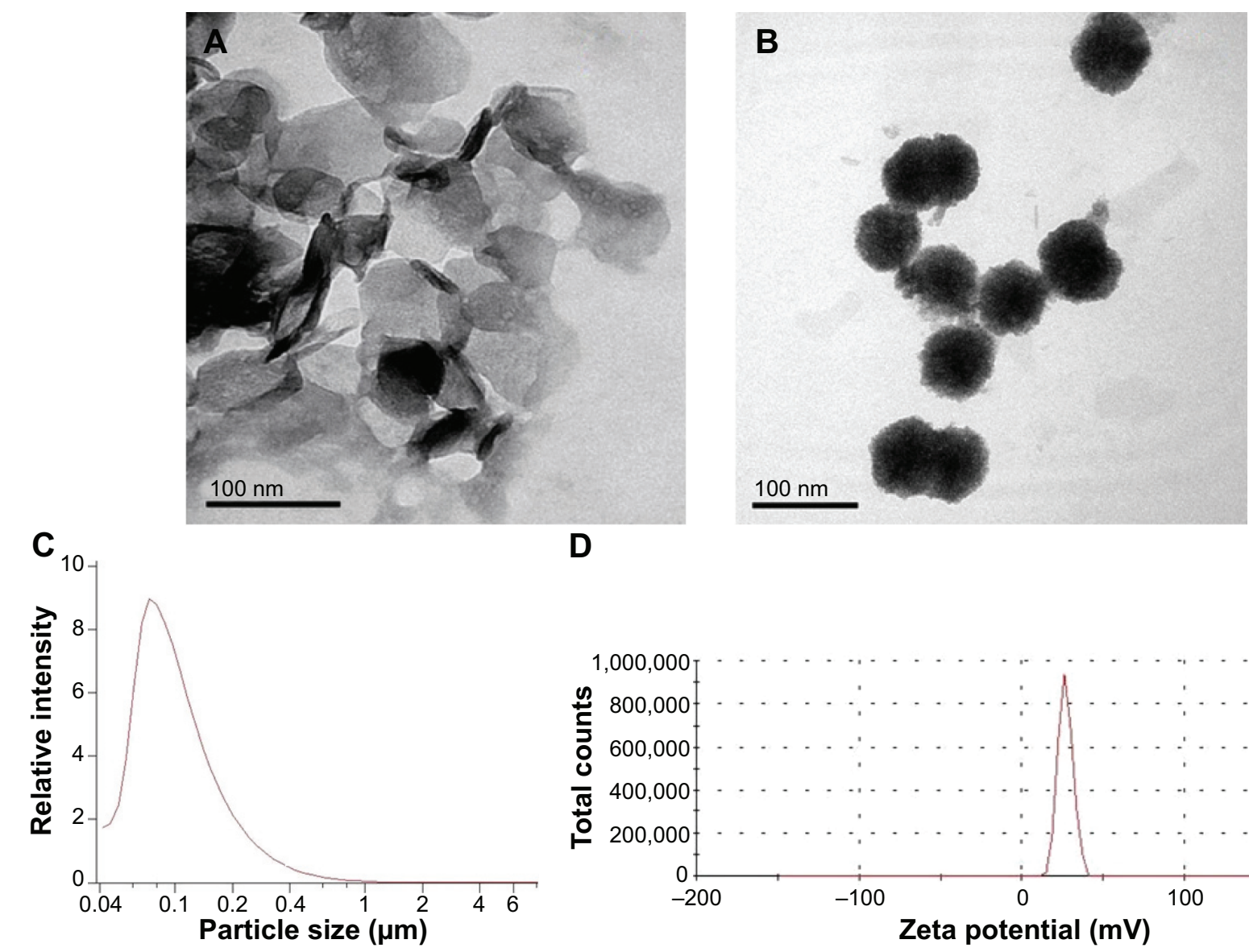

D

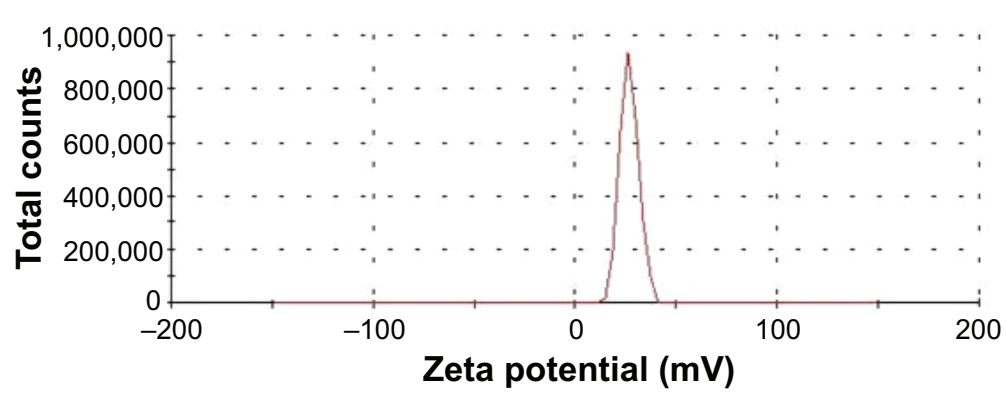

Figure 3 TEM images of nanohybrids.

Notes: (A) LDH crystals (B) folic acid-LDH nanohybrid crystals. Particle size distribution (C) and zeta potential distribution (D) of folic acid-LDH nanoparticles. Abbreviations: LDH, layered double hydroxide; TEM, transmission electron microscope.

folic acid loaded into LDH exhibits an obvious improved antioxidant capacity in comparison with the same concentration of free-drug folic acid. Therefore, folic acid-LDH nanoparticles possess in vitro antioxidant abilities that imply

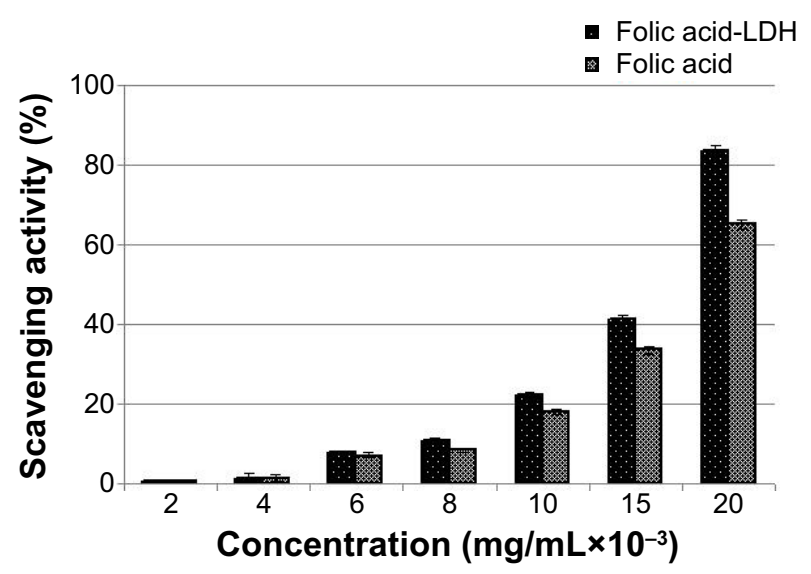

Figure 4 Hydroxyl radical scavenging activity of folic acid and folic acid-LDH. Note: Results represent the means of three independent experiments. Abbreviation: LDH, layered double hydroxide. their potential to reduce the lipid peroxidation and reinforce antioxidant defense of free radicals. Moreover, they may have the potential ability to reduce oxidative stress in vivo and protect the body from fatigue. ${ }^{32}$

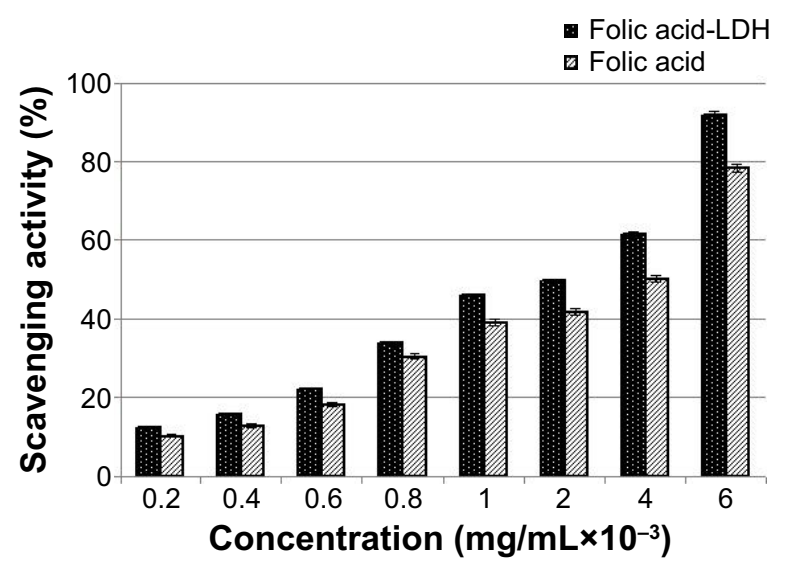

Figure 5 DPPH radical scavenging activity of folic acid and folic acid-LDH. Note: Results represent the means of three independent experiments. Abbreviations: DPPH, I, I-diphenyl-2-picrylhydrazyl; LDH, layered double hydroxide. 


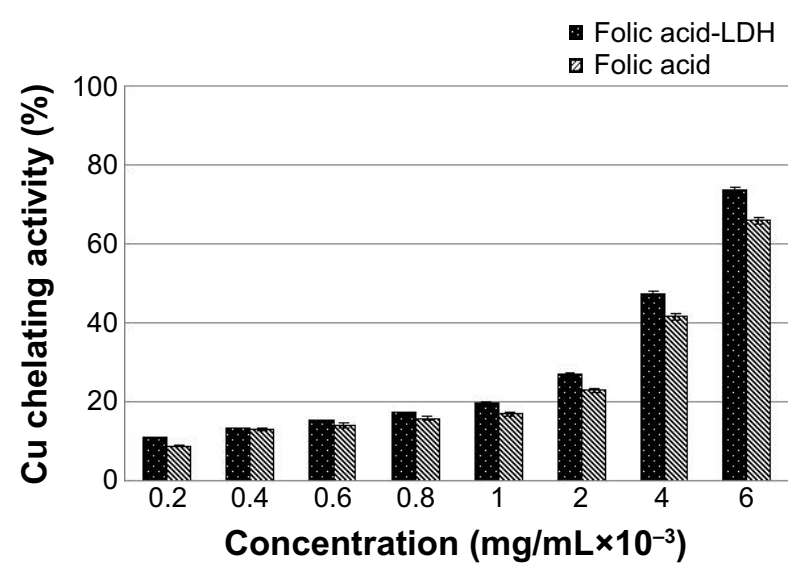

Figure 6 Cupric ion chelating activity of folic acid and folic acid-LDH. Note: Results represent the means of three independent experiments. Abbreviation: LDH, layered double hydroxide.

\section{Cytotoxicity study of folic acid-LDH}

The cytotoxicity of pure folic acid and folic acid-LDH were evaluated in GES-1 using MTT tetrazolium assay. The yellow tetrazolium MTT was reduced by metabolically active cells, in part by the action of hydrogenase enzymes to generate reducing equivalents such as NADH and NADPH. The resulting intracellular purple formazan was solubilized and quantized by spectrophotometric means. Figure 7 shows the effect of folic acid and folic acid-LDH on the proliferation of GES-1 cells at various concentrations. Both pure drug folic acid and folic acid-LDH nanoparticles do not show an obvious toxicity to GES-1 cells owing to the fact that the rate of living cells is maintained at $90 \%$ after incubation for 48 hours at each of the tested concentrations. Therefore, this confirms that folic acid-LDH is hardly toxic to GES-1 cells and can serve as a safe drug-delivery system in the cellular system.

\section{In vivo antifatigue capacity of folic acid-LDH}

Effect of folic acid-LDH on prolonged swimming time The forced swimming test is one of the most commonly used animal models for evaluating physical endurance capacity as it provides high reproducibility. Reduced susceptibility to fatigue is correlated with longer swimming times. ${ }^{33}$ The forced swimming time of mice is shown in Figure 8. Compared to the control groups, oral administration of both folic acid and folic acid-LDH significantly $(P<0.05)$ prolong the time to exhaustion. The average swimming time of the folic acidLDH-mice group was 13.12 minutes, which is an increase of $51 \%$ compared with the control group. Meanwhile, the forced swimming time of the folic acid groups was 9.42 minutes,

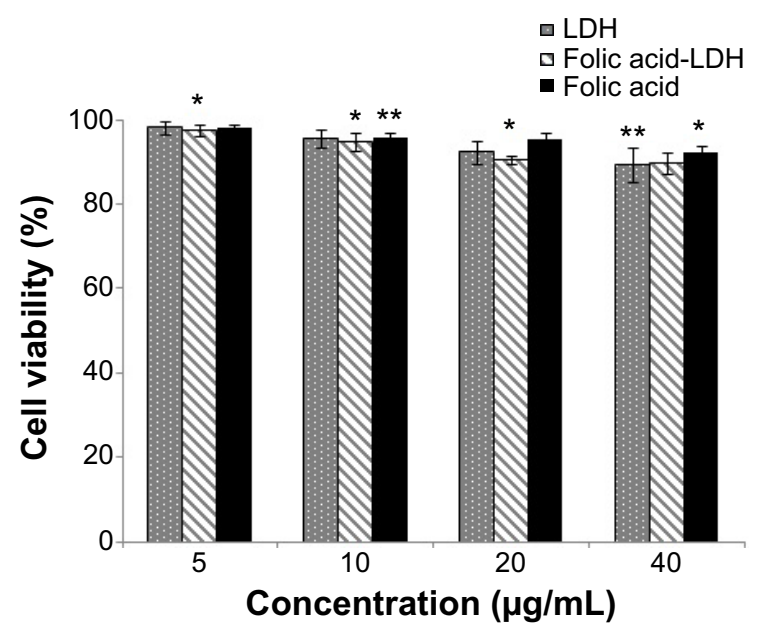

Figure 7 Cell viability of LDH, folic acid, and folic acid-LDH on the growth of GES-I cells.

Notes: The cells have been treated with one of these three agents for 48 hours at concentrations of $5,10,20$, and $40 \mu \mathrm{g} / \mathrm{mL}$. Results represent the means of three independent experiments and error bars represent the standard error of the mean. $\mathrm{N}=3, * P<0.05 ; * * P<0.001$. All entries showed significant difference with the control group according to the Student's $t$-test.

Abbreviation: LDH, layered double hydroxide.

which is $32 \%$ longer than that of the control groups. The swimming-time data suggest that the administration of folic acid-LDH could prolong the swimming time for exhaustion, indicating that folic acid-LDH possesses an antifatigue effect that could elevate exercise endurance. ${ }^{34}$

\section{Effect of biochemical parameters related to fatigue}

BUN is a product of energy metabolism representing normally kidney function, which is the metabolism outcome of protein and amino acid fatigue. ${ }^{35}$ Urea as the end product of protein metabolism and is formed in the liver. In the process

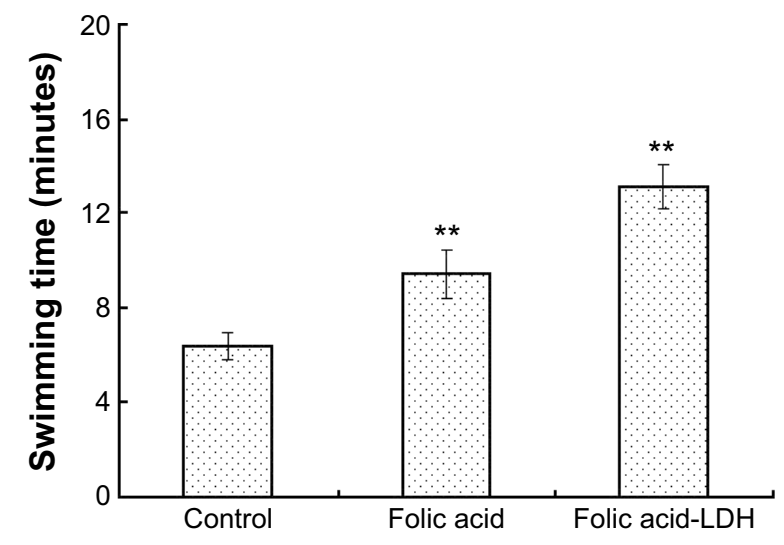

Figure 8 Effect of folic acid and folic acid-LDH on the swimming time in mice. Notes: Results present means of three independent experiments and error bars represent the standard error of the mean. $\mathrm{N}=3, * * P<0.00 \mathrm{I}$. All entries showed significant difference compared with the control group.

Abbreviation: LDH, layered double hydroxide. 
of digestion, protein is broken down into small peptides and amino acid. The amino acid nitrogen is removed as $\mathrm{NH}_{4}^{+}$, and the rest of the molecule is used to produce energy or other substances for the cell demand. ${ }^{36}$ As BUN is a sensitive index of fatigue status, it has a positive correlation between urea nitrogen in vivo and exercise tolerance, which means that the worse the body is adapted for exercise tolerance, the more significantly the urea nitrogen level increases. ${ }^{37}$ As shown in Figure 9, the levels of BUN in folic acid and folic acid-LDH groups are lower than those in control groups. The BUN contents in the folic acid groups were significantly lower by $10.0 \%$ compared to the control groups, while those in the folic acid-LDH groups were decreased by $19.1 \%$, indicating that folic acid-LDH may reduce protein metabolism and enhance endurance. ${ }^{38}$

BLA is the glycolysis product of carbohydrates under an anaerobic condition. It results from muscles doing highintensity exercise in a short time. The excess lactic acid can be removed and converted to glucose via a gluconeogenesis pathway, then the excess glucose will be stored as hepatic glycogen. ${ }^{39}$ With the intense exercises increased, the higher level of lactic acid will cause an increased $\mathrm{H}^{+}$concentration and change the internal $\mathrm{pH}$ value. It will induce many negative side effects on cells, organs, and physiological processes that ultimately result in fatigue. ${ }^{40}$ Therefore, the blood lactate acid is one of the important indexes to reflect the fatigue degree. As per the results shown in Figure 9, it is found that folic acid and folic acid-LDH can reduce the BLA level by $21.3 \%$ and $30.5 \%$, respectively. The results indicate that folic acid-LDHs have a better effect on reducing BLA and can protect the body from appearance of fatigue.

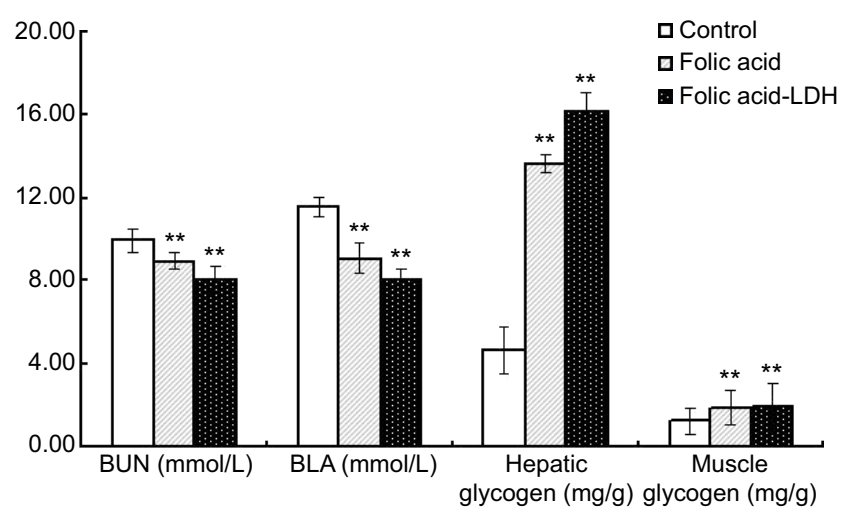

Figure 9 Effect of folic acid and folic acid-LDH on BUN, BLA, hepatic glycogen, and muscle glycogen contents of mice.

Notes: Results represent means of three independent experiments and error bars represent the standard error of the mean. $\mathrm{N}=3, * * P<0.001$ as compared with the control group.

Abbreviations: BLA, blood lactate acid; BUN, blood urea nitrogen; LDH, layered double hydroxide.
It has been established that the effect of energy stored as glycogen is one of the most important factors in determining the capacity for prolonged exercise. ${ }^{41}$ When muscle glycogen stores are depleted during exercise, "the content of glycogen stores lasts longer in liver than in muscle. ${ }^{42}$ Energy for exercise is derived initially from the utilizing of glycogen, then from the glycogen stored in liver, and later from non-esterified fatty acids. ${ }^{43}$ Therefore, liver and muscle glycogen are sensitive indices related to fatigue. The data in Figure 9 reveals that the levels of hepatic glycogen and muscle glycogen in both folic acid and folic acid-LDH groups are much higher than those in control groups. The folic acid-LDH groups show an obvious increased content level compared to the folic acid groups. The data indicates that folic acid-LDH can increase the storage of hepatic glycogen leading to enhanced exercise endurance and improved energy metabolism activity.

Many studies have reported that folic acid as a vitamin of the B group reacts with the free radicals that play an important role on antioxidant effect. ${ }^{44}$ The in vivo antifatigue assays indicate that both folic acid and folic acid-LDH groups showed obvious increased effects on biochemical parameters related to fatigue compared to the control groups. Moreover, folic acid intercalates into LDH delivery are more effective in decreasing BUN and BLA, and increasing muscle and hepatic glycogen level compared to the folic acid groups. We propose the following mechanism: firstly, the LDH nanomaterials have a sustained release profile which can help increase the practical delivery of folic acid and the drug molecules. The folic acid is more stabilized when it is entrapped in LDH, which can protect it from degradation in the biochemical reaction process. ${ }^{17}$ Secondly, loading LDH nanoparticles with antioxidants would improve cellular drug uptake and retention. ${ }^{45,46}$ Therefore, the folic acid-LDH delivery system may be used as a potential antioxidant supplement for a broad range of exercise endurance and antifatigue applications. This research provides an important basis for developing nanomaterial nutritional supplements suitable for antioxidant and antifatigue effects.

\section{Conclusion}

In this research, the folic acid-LDHs were successfully prepared with $32.4 \%$ (w/w) drug loading. The composition, structure, particle shape, and size of the nano-antioxidants were also characterized. The in vitro antioxidant effect assays demonstrate that folic acid-LDH antioxidant systems have an obvious DPPH scavenging effect, hydroxyl radicals scavenging effect, and pro-oxidative $\mathrm{Cu}^{2+}$ chelating effect. The in vitro cytotoxicity assays indicate that the folic 
acid-LDH antioxidant system has no significant cytotoxic effect or obvious toxicity to normal cells. The in vivo antifatigue study shows that folic acid-LDH can prolong the forced swimming time of the mice. Folic acid-LDH nanomaterials are more effective in decreasing BUN and BLA, and increasing muscle and hepatic glycogen levels compared to folic acid groups and control groups. These results imply that the folic acid-LDH antioxidant system increases the endurance capacity and facilitates recovery from fatigue. They indicate that it may be used as a novel antioxidant or antifatigue nutritional supplement. Future work will focus on the study of antioxidant and antifatigue mechanisms at the molecular level.

\section{Acknowledgments}

This work was financially supported by the National Natural Science Foundation of China (Grant No 31100855, 31260251, 81301310), a Special Financial Grant from the China Postdoctoral Science Foundation (Grant No 2012T50440).

\section{Disclosure}

The authors report no conflicts of interest in this work.

\section{References}

1. Tsakiris S, Parthimos T, Parthimos N, et al. The beneficial effect of L-cysteine supplementation on DNA oxidation induced by forced training. Pharmacol Res. 2006;53:386-390.

2. Takenaka A, Annaka H, Kimura Y, Aoki H, Igarashi K. Reduction of paraquat-induced oxidative stress in rats by dietary soy peptide. Biosci Biotechnol Biochem. 2003;67:278-283.

3. Elia D, Stadler K, Horváth V, Jakus J. Effect of soy- and whey proteinisolate supplemented diet on the redox parameters of trained mice. Eur J Nutr. 2006;45:259-266.

4. Naidu MM, Shyamala BN, Pura Naik J, Sulochanamma G, Srinivas P. Chemical composition and antioxidant activity of the husk and endosperm of fenugreek seeds. LWT - Food Science and Technology. 2011;44:451-456.

5. Kirwan JP, O'Gorman D, Evans WJ. A moderate glycemic meal before endurance exercise can enhance performance. J Appl Physiol (1985). 1998;84(1):53-59.

6. Joshi R, Adhikari S, Patro BS, Chattopadhyay S, Mukherjee T. Free radical scavenging behavior of folic acid: evidence for possible antioxidant activity. Free Radic Biol Med. 2001;30:1390-1399.

7. Racek J, Rusnáková H, Trefil L, Siala KK. The influence of folate and antioxidants on homocysteine levels and oxidative stress in patients with hyperlipidemia and hyperhomocysteinemia. Physiol Rev. 2005;54:87-95.

8. Bayés B, Pastor MC, Bonal J, Juncà J, Romero R. Homocysteine and lipid peroxidation in hemodialysis: role of folinic acid and vitamin $\mathrm{E}$. Nephrol Dial Transplant. 2001;16:2172-2175.

9. Xu ZP, Lu GQ. Layered double hydroxide nanomaterials as potential cellular drug delivery agents. Pure and Applied Chemistry. 2006;78:1771-1779.

10. Xu ZP, Zeng QH, Lu GQ, Yu AB. Inorganic nanoparticles as carriers for efficient cellular delivery. Chem Eng Sci. 2006;61:1027-1040.

11. Li A, Qin LL, Wang WR, et al. The use of layered double hydroxides as DNA vaccine delivery vector for enhancement of anti-melanoma immune response. Biomaterials. 2011;32:469-477.
12. Xu ZP, Walker TL, Liu KL, Cooper HM, Lu GQ, Bartlett PF. Layered double hydroxide nanoparticles as cellular delivery vectors of supercoiled plasmid DNA. Int J Nanomedicine. 2007;2:163-174.

13. Zhang H, Qi R, Evans DG, Duan X. Synthesis and characterization of a novel nano-scale magnetic solid base catalyst involving a layered double hydroxide supported on a ferrite core. J Solid State Chem. 2004;177:772-780.

14. Hayashi A, Fujimoto Y, Ogawa Y, Nakayama H, Tsuhako M. Adsorption of gaseous formaldehyde and carboxylic acids by ammoniumion-exchanged alpha-zirconium phosphate. J Colloid Interface Sci. 2005;283:57-63.

15. Zhang H, Zou K, Guo S, Duan X. Nanostructural drug-inorganic clay composites: Structure, thermal property and in vitro release of captoprilintercalated Mg-Al-layered double hydroxides. J Solid State Chem. 2006; 179:1792-1801.

16. Qin LL, Xue M, Wang WR, et al. The in vitro and in vivo anti-tumor effect of layered double hydroxides nanoparticles as delivery for podophyllotoxin. Int J Pharm. 2010;388:223-230.

17. Tyner KM, Schiffman SR, Giannelis EP. Nanobiohybrids as delivery vehicles for camptothecin. J Control Release. 2004;95:501-514.

18. Li B, He J, Evans DG, Duan X. Enteric-coated layered double hydroxides as a controlled release drug delivery system. Int $J$ Pharm. 2004;287:89-95.

19. Xiao R, Wang WR, Pan L, et al. A sustained folic acid release system based on ternary magnesium/zinc/aluminum layered double hydroxides. J Mater Sci. 2011;46:2635-2643.

20. Qin L, Wang S, Zhang R, Zhu R, Sun X, Yao S. Two different approaches to synthesizing Mg-Al-layered double hydroxides as folic acid carriers. J Phys Chem Solids. 2008;69:2779-2784.

21. de Avellar IG, Magalhães MM, Silva AB, Souza LL, Leitão AC, Hermes-Lima M. Reevaluating the role of 1,10-phenanthroline in oxidative reactions involving ferrous ions and DNA damage. Biochim Biophys Acta. 2004;1675:46-53.

22. Shimada K, Fujikawa K, Yahara K, Nakamura T. Antioxidative properties of xanthan on the autoxidation of soybean oil in cyclodextrin emulsion. J Agr Food Chem. 1992;40:945-948.

23. Zhu L, Chen J, Tang X, Xiong YL. Reducing, radical scavenging, and chelation properties of in vitro digests of alcalase-treated zein hydrolysate. J Agr Food Chem. 2008;56:2714-2721.

24. Tan W, Yu KQ, Liu YY, et al. Anti-fatigue activity of polysaccharides extract from Radix Rehmanniae Preparata. Int J Biol Macromol. 2012; 50:59-62.

25. Wei M, Xu X, Wang X, et al. Study on the photochromism of Ni-Al layered double hydroxides containing nitrate anions. Eur J Inorg Chem. 2006;14:2831-2838.

26. Choy JH, Jung JS, Oh JM, et al. Layered double hydroxide as an efficient drug reservoir for folate derivatives. Biomaterials. 2004;25: 3059-3064.

27. Wang W, Zhu R, Xie Q, et al. Enhanced bioavailability and efficiency of curcumin for the treatment of asthma by its formulation in solid lipid nanoparticles. Int J Nanomedicine. 2012;7:3667-3677.

28. Li Y, Jiang B, Zhang T, Mu W, Liu J. Antioxidant and free radicalscavenging activities of chickpea protein hydrolysate $(\mathrm{CPH})$. Food Chem. 2008;106:444-450.

29. Lipinski B. Hydroxyl radical and its scavengers in health and disease. Oxid Med Cell Longev. 2011;2011:809696.

30. Jao CL, Ko WC. 1,1-dipheny1-2-picrylhydrazyl (DPPH) radical scavenging by protein hydrolyzates from Tuna cooking juice. Fisheries Sci. 2002;68:430-435.

31. Yu L, Haley S, Perret J, Harris M, Wilson J, Qian M. Free radical scavenging properties of wheat extracts. J Agr Food Chem. 2002;50: $1619-1624$.

32. Bajpai VK, Sharma A, Kang SC, Baek KH. Antioxidant, lipid peroxidation inhibition and free radical scavenging efficacy of a diterprenoid compound sugiol isolated from Metasequoia glyptostroboides. Asian Pac J Trop Med. 2014;7:9-15. 
33. Jung K, Kim IH, Han D. Effect of medicinal plant extracts on forced swimming capacity in mice. J Ethnopharmacol. 2004;93:75-81.

34. Kumar GP, Anand T, Singsit D, Khanumb F, Anilakumar KR. Evaluation of antioxidant and anti-fatigue properties of Trigonella foenum-graecum L. in rats subjected to weight loaded forced swim test. Pharmacognosy Journal. 2013;5:66-71.

35. Wang JJ, Shieh MJ, Kuo SL, Lee CL, Pan TM. Effect of red mold rice on antifatigue and exercise-related changes in lipid peroxidation in endurance exercise. Appl Microbiol Biotechnol. 2006;70:247-253.

36. Koo HN, Lee JK, Hong SH, Kim HM. Herbkines increases physical stamina in mice. Biol Pharml Bull. 2004;27(1):117-119.

37. Zhang Y, Yao X, Bao B, Zhang Y. Anti-fatigue activity of a triterpenoidrich extract from Chinese bamboo shavings (Caulis bamfusae in taeinam). Phytother Res. 2006;20:872-876.

38. Wang L, Zhang HL, Lu R, et al. The decapeptide CMS001 enhances swimming endurance in mice. Peptides. 2008;29:1176-1182.

39. Robergs RA, Ghiasvand F, Parker D. Biochemistry of exerciseinduced metabolic acidosis. Am J Physiol Regul Integr Comp Physiol. 2004;287:R502-R516.
40. Jia JM, Wu CF. Anti fatigue activity of tissue culture extracts of Saussurea involucrate. Pharm Biol. 2008;46:433-436.

41. Jung KA, Han D, Kwon EK, Lee CH, Kim YE. Antifatigue effect of Rubbus coreanus Miquel extract in mice. J Med Food. 2007; 10:689-693.

42. Terjung RL, Baldwin KM, Molé PA, Klinkerfuss GH, Holloszy JO. Effect of running to exhaustion on skeletal muscle mitochondria: A biochemical study. Am J Physiol. 1972;223:549-554.

43. Dorchy H. [Sports and type I diabetes: personal experience]. Rev Med Brux. 2002;23:A211-A217. French.

44. Burton GW, Ingold KU. beta-Carotene: an unusual type of lipid antioxidant. Science. 1984;224:569-573.

45. Vanaja K, Wahl MA, Bukarica L, Heinle H. Liposomes as carriers of the lipid soluble antioxidant resveratrol: evaluation of amelioration of oxidative stress by additional antioxidant vitamin. Life Sci. 2013;93: 917-923.

46. Choy JH, Kwak SY, Park JS, Jeong YJ. Cellular uptake behavior of $\left[\gamma^{32} \mathrm{P}\right]$ labeled ATP-LDH nanohybrids. J Mater Chem. 2001;11:1671-1674.
International Journal of Nanomedicine

\section{Publish your work in this journal}

The International Journal of Nanomedicine is an international, peerreviewed journal focusing on the application of nanotechnology in diagnostics, therapeutics, and drug delivery systems throughout the biomedical field. This journal is indexed on PubMed Central, MedLine, CAS, SciSearch ${ }^{\circledR}$, Current Contents ${ }^{\circledR} /$ Clinical Medicine,

\section{Dovepress}

Journal Citation Reports/Science Edition, EMBase, Scopus and the Elsevier Bibliographic databases. The manuscript management system is completely online and includes a very quick and fair peer-review system, which is all easy to use. Visit http://www.dovepress.com/ testimonials.php to read real quotes from published authors. 